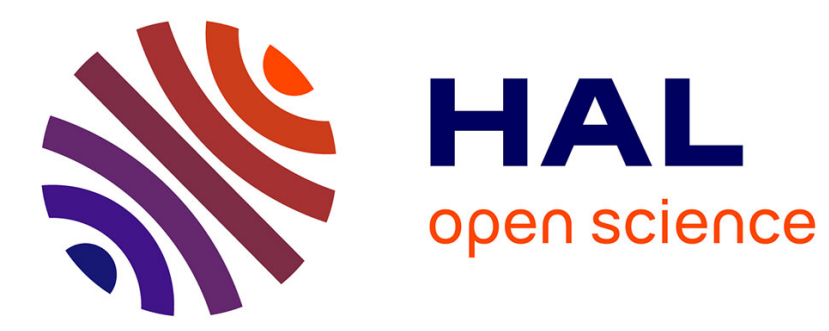

\title{
L'économie entre performativité, idéologie et pouvoir symbolique
}

\author{
Bruno Ambroise, Grégory Salle, Richard Sobel
}

\section{To cite this version:}

Bruno Ambroise, Grégory Salle, Richard Sobel. L'économie entre performativité, idéologie et pouvoir symbolique. L'Homme et la Société, 2015, 197 (3), pp. 13-30. halshs-02088109

\section{HAL Id: halshs-02088109 \\ https://shs.hal.science/halshs-02088109}

Submitted on 2 Apr 2019

HAL is a multi-disciplinary open access archive for the deposit and dissemination of scientific research documents, whether they are published or not. The documents may come from teaching and research institutions in France or abroad, or from public or private research centers.
L'archive ouverte pluridisciplinaire $\mathbf{H A L}$, est destinée au dépôt et à la diffusion de documents scientifiques de niveau recherche, publiés ou non, émanant des établissements d'enseignement et de recherche français ou étrangers, des laboratoires publics ou privés. 


\section{L'économie entre performativité, idéologie et pouvoir symbolique}

Il est devenu presque banal de remarquer que, depuis bientôt deux décennies, la « performativité » est devenue une notion en vogue dans les sciences sociales. Le succès était déjà suffisamment notable pour qu'un tel constat soit, il y a dix ans, le point de départ d'une analyse des «migrations » de cette notion, passant en revue ses «nouveaux visages ' ». Un succès au demeurant assez paradoxal si l'on considère que, comme l'ont remarqué eux-mêmes certains des initiateurs de ce remaniement conceptuel, il s'agissait chez son créateur, John L. Austin'2, d'une notion problématique et même caduque, dans la mesure où il s'est évertué à la dépasser à peine élaborée. Il s'agissait en outre d'une notion qui ne prenait pleinement sens qu'au sein de sa réflexion générale sur les actions accomplies au moyen du langage et des problèmes auxquels elle répondait. Pourtant, elle s'est répandue d'une manière si manifeste qu'on a pu parler d'un « tournant performatif ${ }^{3}$ » dans les sciences sociales en général et dans la sociologie économique et l'épistémologie de l'économie en particulier, tournant amorcé par l'ouvrage collectif inaugural publié en

1. Jérôme DenIS, «Préface : Les nouveaux visages de la performativité », Études de communication, $\mathrm{n}^{\circ} 29,2006$, p. 8-24. Voir plus largement le dossier «Performativité : relectures et usages d'une notion frontière » dont ce texte est l'introduction, notamment l'article de Marc LENGLET, «Des paroles aux actes : usages contemporains de la performativité dans le champ financier». Deux ans plus tard, le colloque «Performativité et politique : au-delà de la sociologie économique », organisé à l'université de Toulouse en octobre 2008, témoignait de la vivacité de cette approche.

2. John L. Austin, Quand dire, c'est faire, Paris, Seuil, 1991 [orig. : How to do things with words?,1962].

3. Voir Fabian MuniesA, The Provoked Economy. Economic Reality and the Performative Turn, Londres/New York, Routledge, 2014 (notamment la $1^{\text {r }}$ partie, p. 5 sqq.). L'expression se retrouve dans une perspective propre chez Christian LicopPE, « Michel Callon et le "tournant performatif" de la théorie de l'acteur-réseau », in Madeleine AKrich, Yannick Barthe, Fabian Muniesa et Philippe Mustar (dir.), Débordements. Mélanges offerts à Michel Callon, Paris, Presses des Mines, 2010, p. 291-298.

L'homme et la société, $\mathrm{n}^{\circ} 197$, juillet-septembre 2015 
1998 sous la direction de Michel Callon ${ }^{4}$. Reprise et discutée par d'autres auteurs, en France mais aussi, voire surtout, dans le monde anglophone, cette approche s'est développée en tant que contribution importante au renouvellement théorico-empirique des sciences sociales de l'économie, justifiant par exemple un chapitre dans le Traité de sociologie économiques.

Des débats ont eu lieu depuis la fin des années 1990 quant à la précision conceptuelle et la portée heuristique de cette notion et donc à l'envergure de l'innovation théorique auquel elle prétendait ${ }^{\circ}$. Toutefois, ces débats, même s'ils furent parfois assez vifs, ont été partiellement éclipsés par l'ampleur et la rapidité de la diffusion du terme de "performativité » et ses dérivés, bien au-delà d'ailleurs du domaine économique. De plus, en dépit d'efforts de clarification de la part de ses introducteurs et promoteurs, qui ont pu lever certains malentendus, le terme pâtit d'un effet de mode qui contribue à nourrir l'équivoque, quand il n'est pas utilisé à tort et à travers. Le fait que, ces dernières années, une expression quelque peu brumeuse comme celle de «critical performativity » ait fait son chemin du côté des dites «sciences de gestion» signale que, sous couvert de prolongements ou de compléments, on risque fort une dilution conceptuelle semant la confusion plutôt qu'apportant un quelconque éclaircissement. Il en va de même, dans une moindre mesure, avec l'expression de «contre-performativité » dont certains usages au moins peuvent laisser perplexe. Il n'est pas superflu d'évoquer ici un décalage ou un hiatus linguistique rarement relevé, qui pourtant contribue sans doute à nourrir des mésententes transatlantiques. En français les mots construits ou traduits autour de la racine «perform-» (le verbe « performer » ou le substantif «performation », que M. Callon a substitué dans un second temps à «performativité »') véhiculent une connotation

4. Michel CALlon (dir.), The Laws of the Markets, Oxford, Blackwell, 1998.

5. Fabian Muniesa et Michel CALLON, «La performativité des sciences économiques », in Philippe STEINER et François VATIN (dir.), Traité de sociologie économique, Paris, PUF, 2009, chap. 8, p. 289-324.

6. De ce point de vue, l'ouvrage de Donald MacKenzie, Fabian Muniesa et Lucia SiU (eds.), Do Economists Make Markets? On the Performativity of Economics, Princeton, Princeton University Press, 2007 apparaît autant, voire plus, comme un état des lieux des désaccords que comme un manifeste.

7. Voir la formulation des «tensions constitutives », in Fabian MunIESA et Michel CAllon, «La performativité des sciences économiques », op. cit., p. 297-302. D’où la traduction utilisée par Donald MACKenZIE et Yuval MiLlo, «Construction d'un marché et performation théorique. Sociologie historique d'une bourse de produits dérivés financiers », Réseaux, $\mathrm{n}^{\circ} 122,2003$, p. 15-61. 
quelque peu ésotérique et en tout cas savante (ces termes n'apparaissent pas dans les dictionnaires courants) qu'elle n'a pas en anglais, où to perform est un verbe parfaitement commun, qui se traduit par « effectuer », « réaliser» voire tout simplement «faire». De ce point de vue, même une fois posé que «[p]erformer c'est provoquer, instaurer, constituer, faire que quelque chose a lieu ${ }^{8} »$, le surplus de précision théorique obtenu par l'usage du terme de «performation» par rapport à « réalisation », «effectuation» ou même «actualisation» (au sens du passage de la puissance à l'acte) n'est pas toujours évident.

Ce sont là de premières raisons justifiant de verser une nouvelle pièce, au moyen de ce numéro de L'homme et la société, à un dossier déjà raisonnablement épais. Mais la raison décisive réside dans la conjoncture théorico-politique qui est la nôtre - qu'on ose à peine qualifier de « crise » tant le mot a perdu tout sens hormis la désignation d'un mode de gouvernement ${ }^{~}$. On sait que la notion d' « épreuve » figure en bonne place dans la panoplie conceptuelle de la «sociologie de la traduction» (ou «théorie de l'acteur-réseau »), dont «l'approche par le performatif » est une expression tardive. Or, on conçoit difficilement épreuve de réalité plus massive que la «crise » ouverte en 2007-2008. Convertie en épreuve de connaissance, elle offre d'autant plus un test empirique grandeur nature aux efforts théoriques que l'une de ses caractéristiques est précisément d'avoir entraîné un vaste ensemble de réflexions sur l'impact des « idées » sur le « réel », y compris sous la forme documentaire ${ }^{10}$. En quoi alors la sociologie de la traduction, via la notion de performativité telle qu'elle l'emploie, aide-t-elle concrètement à penser une « crise » (guillemets inclus) qui fait directement écho aux préoccupations qu'elle a mises en avant?

L'enjeu tient en somme en un triple questionnement. Le premier est celui des conditions de pertinence de l'extension sémantique d'un concept extrait de sa problématique initiale : l'identité formelle du mot peut masquer un usage qui a peu à voir avec son acception première, ce qui, même si la torsion de sens est parfois signalée par ceux qui l'opèrent, est source de confusion dans la mesure où le terme est de fait attaché à

8. Fabian Muniesa et Michel CALlon, «La performativité des sciences économiques », op. cit., p. 301 .

9. Voir par exemple le numéro 30 de la revue Lignes (2009), « De la crise comme méthode de gouvernement ».

10. On pense notamment à The Inside Job (Charles FERGuSON, 2010) ou Capitalisme (Ilan ZIV, 2014). 
Austin qui l'a construit pour des raisons conceptuelles précises. Le deuxième concerne la portée heuristique de la notion : dans quelle mesure a-t-elle été source de connaissance ou, en d'autres termes, a-t-elle fondé un regard réellement original sur les objets concernés, mettant en lumière une dimension jusque-là négligée ou inaperçue ? Le troisième questionnement, le principal peut-être, a trait au rapport entre l'approche par la performativité telle que portée par la sociologie de la traduction, d'une part, et d'autres courants de pensée et perspectives théoriques, de l'autre, et ce particulièrement du point de vue de la question de la critique. La position de cette sociologie paraît de ce point de vue ambivalente, hésitant entre critique de la critique, posture post-critique et reprise de la critique par d'autres moyens; une oscillation que la conjoncture présente invite à discuter.

\section{Un emprunt terminologique qui produit des effets heuristiques au risque d'un brouillage théorique? (ou: un enfant dans le dos de John L. Austin)}

Élaborée à l'origine dans le cadre de la philosophie du langage ordinaire (contre le paradigme descriptif du langage), puis dans la pragmatique linguistique (davantage attentive aux conditions langagières d'énonciation), la notion de performativité a vu son sens s'élargir à mesure que son usage s'est diffusé à divers champs des sciences sociales. On la retrouve en effet de la philosophie des normes - ce qui n'est guère étonnant au regard de la connexion originaire entre actes de langage et droit, personnifiée non seulement par Austin mais aussi par Adolf Reinach" - jusqu'à l'anthropologie de l'écriture, en passant par la théorie sociale et notamment les gender studies ${ }^{\prime 2}$. Mais c'est sans doute dans la sociologie économique que son succès est le plus patent. Son adoption a donné lieu à un ensemble de travaux stimulants, y compris par certaines différences entre les auteurs sur certains aspects (relatifs par exemple à la production de l'homo ceconomicus et à la notion d'encastrement), travaux appuyés sur de nombreux cas d'étude

11. Pour une discussion autour de ce point, voir, dans deux perspectives différentes, Sandra LAUGieR, "Performativité, normativité et droit», Archives de Philosophie, $n^{\circ} 67 / 4$, p. 607-627 ; Béatrice FRAENKEL, « Actes écrits, actes oraux : la performativité à l'épreuve de l'écriture », Études de communication, n² 29, 2006, p. 69-93.

12. Voir Judith ButLer, Le pouvoir des mots. Politique du performatif, Paris, éd. Amsterdam, 2004. Pour une critique de cette conception, voir Bruno AMBroISE, « Peut-on penser une construction performative du genre ? », Agone, $\mathrm{n}^{\circ}$ 43, 2010, p. 142-167. 
permettant de voir la notion à l'œuvre dans des analyses empiriques. C'est même sans doute l'un des principaux mérites de cette approche que d'avoir constitué un véritable programme théorico-empirique (contre la fétichisation du «terrain» ou la survalorisation des «usages»), se servant d'un effort de remaniement conceptuel comme d'un aiguillon de l'enquête.

Par-delà des variations ou distinctions mineures, l'idée fondamentale est la suivante : les «sciences économiques ", l'économie comme discipline, comme savoir, ne forment pas seulement (voire pas du tout) un discours neutre se bornant à décrire ou observer l'économie comme activité ; bien plus, elles la «performent», c'est-à-dire qu'elles contribuent à la faire advenir, qu'elles participent à son accomplissement, à sa configuration, à son objectivation. En ce sens, on a pu considérer que, à l'instar des discours qui modifient l'état du monde, tel celui de la promesse ou celui du mariage, le discours économique avait un effet «performatif ». Cette idée maîtresse n'apparaît cependant pas comme l'apport le plus original, même s'il est vrai qu'elle éclaire à sa manière le point aveugle d'une position consistant simplement à dévoiler les impensés et à dénoncer l'irréalisme, sinon la fausseté mystificatrice, des modélisations économiques ${ }^{3}$. Reconnaître qu'un discours (à prétention) scientifique a des effets observables dans le monde réel n'a en soi rien de très nouveau. Ce ne serait pas faire beaucoup crédit aux penseurs du capitalisme que de penser qu'ils n'avaient jamais songé au fait que, par exemple, les conceptions mercantilistes ou physiocratiques (et plus tard monétaristes ou keynésiennes) ont puissamment informé le capitalisme « réel »... Cela ne s'est pas toujours accompagné de descriptions en bonne et due forme, certes... Quoique : plus près de nous, en s'opposant au paradigme descriptif du langage statistique pour en montrer tout l'aspect normatif voire prescriptif, Alain Desrosières n'avait-il pas, non seulement dit, mais bien montré l'essentiel ? ${ }^{14}$ On y retrouvait en tout cas un enjeu central de la sociologie de la traduction, consistant à suivre pas à pas comment des énoncés sont traduits en modes d'organisation pratiques, par quelle chaîne

13. Sur la critique sociologique de l'économie comme «fausse science», voir Philippe STEINER, «La tradition française de critique sociologique de l'économie politique », Revue d'Histoire des Sciences Humaines, ${ }^{\circ} 18,2008$, p. 63-84.

14. Voir les travaux d'Alain Desrosieres, de La politique des grands nombres (Paris, La Découverte, 1993) à Prouver et gouverner (Paris, La Découverte, 2014). Les premiers travaux du britannique Donald MacKenzie, l'un des principaux noms attachés à l'approche considérée ici, avaient justement trait à la sociologie de la statistique. 
sociotechnique on passe du discours (ou d'ailleurs de la pensée) à sa réalisation tangible.

De cette attention à la trame sociotechnique découle sans doute le point le plus remarquable de la sociologie qu'on peut dire, faute de mieux, «performativiste »: l'attention portée non seulement au discours, mais aussi, voire surtout, aux médiations techniques par lesquelles il transite sous forme objectivée, donc aux objets, supports, équipements, etc., jouant un rôle de médiateurs et non seulement d'intermédiaires is. C'est ici que l'usage de la notion se distingue du concept de prophétie autoréalisatrice élaboré par Robert Merton, centrée sur les phénomènes de croyance collective ${ }^{16}$. Les assertions scientifiques ou à prétention scientifique, telles celles de l'économie comme discipline, sont imbriquées dans des rapports non seulement discursifs et plus largement symboliques (juridiques, par exemple) mais aussi dans des dispositifs matériels, l'ensemble formant des agencements ; d'où la propension à parler d'agencements marchands plutôt que de marchés. Si l'étude de ces agencements a donné lieu à de passionnantes études de cas, certaines interrogations subsistent néanmoins. Elles tiennent d'abord au terme directeur lui-même, en raison d'une " opération d'emprunt» qui en a effectivement «modifié le sens » initial ${ }^{1}$. Il faut dire que reprendre un terme forgé en référence au langage pour en fait porter l'attention sur autre chose, et même d'une certaine manière minorer le rôle du discours en se concentrant sur les éléments matériels qui lui donnent consistance, donc s'éloigner fortement de l'inspiration austinienne (pour ne pas dire la prendre à contrepied !), ne pouvait manquer de jeter le trouble... C'est notamment de ce trouble dont traite Nicolas Brisset dans sa contribution au présent dossier intitulée « Comment (et pourquoi) repenser la performativité des énoncés théoriques », contribution qui propose une conception alternative, conventionnaliste, née de l'articulation entre Austin et David Lewis ; pour Austin, en effet, les effets performatifs sont nécessairement conventionnels.

Sans revenir ici en détail sur la genèse et le sens de la notion, abordés dans au moins deux contributions du dossier (celles de Nicolas Brisset et de François Flahault), rappelons qu'Austin forge le terme «d'énoncé

15. Pour reprendre la distinction de Bruno Latour dans L'espoir de Pandore, Paris, La Découverte, 2001, p. 328-329.

16. Michel CALlon, «What Does It Mean to Say That Economics Is Performative ? ", in Donald MacKenzie, Fabian Muniesa et Lucia Siu (eds.), Do Economists Make Markets?, op. cit., p. 321 sq.

17. Fabian MuniESA et Michel CALLON, «La performativité des sciences économiques », op. cit., p. 295. 
performatif » en distinguant d'abord clairement les énoncés constatifs (ou descriptifs) des énoncés performatifs. Les premiers sont les énoncés traditionnellement privilégiés par l'analyse philosophique, en ce qu'ils disent quelque chose à propos du monde et ont ainsi un contenu analysable en termes de conditions de vérité. À l'inverse, les énoncés performatifs identifiés par Austin ne sont précisément pas analysables en termes de vérité ou de fausseté, car il s'avère qu'ils ne décrivent rien mais font plutôt quelque chose ; c'est le cas des énoncés de promesse, de mariage, de baptême, etc. : ces énoncés ne parlent pas de l'état du monde mais le modifient. Or, cette distinction initiale, qui avait surtout pour objet de contester la prééminence accordée à l'analyse logique des énoncés censés dire le vrai, est rapidement battue en brèche par Austin lui-même, qui montre qu'en réalité tout énoncé comprend une dimension «actionnelle » qu'il qualifie d'illocutoire. Autrement dit, la distinction entre les énoncés constatifs et les énoncés performatifs n'était que provisoire et reposait sur l'illusion que seuls certains énoncés avaient une dimension actionnelle. Ayant montré que cette dimension était générale, Austin abandonne la distinction initiale au profit d'une tripartition d'aspects valant pour tous les énoncés : l'aspect locutoire, l'aspect illocutoire et l'aspect perlocutoire. Lorsque l'on parle et que l'on réussit à utiliser ses mots pour dire quelque chose, trois choses au moins, nous dit Austin, sont accomplies : un acte locutoire, un acte illocutoire et un acte perlocutoire. Prenons l'énoncé «Je vous déclare mari et femme », utilisé dans un contexte adéquat et ne subissant pas d'échec. L'acte locutoire consiste à dire que je vous déclare mari et femme ; c'est l'acte d'énonciation signifiant. L'acte illocutoire consiste à vous faire mari et femme, donc à vous marier ; c'est dans cet acte que réside l'effectuation du mariage. Enfin, l'acte perlocutoire est celui de rendre heureux les nouveaux époux, tristes les prétendants éconduits : il correspond aux actes réalisés conséquemment à l'acte illocutoire. Parlant d'acte, Austin entend donc identifier un type d'effet particulier correspondant en définitive à l'acte illocutoire : des effets illocutoires, qui, peut-on considérer, englobent les effets qualifiés initialement par Austin de «performatifs ».

En première analyse, ce dernier avait introduit le terme de performatif dans les années 1940, au cours d'un séminaire avec le philosophe du droit Herbert Hart, pour comprendre le fonctionnement du langage juridique et ainsi analyser d'une nouvelle façon le langage : le droit, en effet, ne se contente pas de dire les choses mais fait bel et bien des choses, sachant qu'Austin allait finalement montrer que tous les types de discours ont ce 
type d'efficacité. Le rapprochement avec l'efficacité des théories économiques semblait donc tentant: dans l'esprit de nos auteurs, la théorie économique ne modifie-t-elle pas le réel comme le droit est capable de le faire ?

Encore faut-il spécifier le type particulier d'effet qu'Austin voulait identifier au moyen du terme «performatif». Une première caractérisation de l'efficacité performative semble être qu'elle fait exister cela même dont elle parle, comme la prononciation d'un ordre ou d'une promesse crée même (ou du moins semble créer) l'ordre ou la promesse. L'économie fait-elle exister en ce sens ce dont elle parle ? C'est une première question. Mais il convient d'ajouter la deuxième caractérisation, décisive : l'efficacité performative porte au jour ce dont un énoncé parle au moyen de la parole elle-même, c'est-à-dire, précisément, en disant telle ou telle chose. C'est en disant - d'une façon ou d'une autre - que l'on promet que l'on promet; c'est en disant que l'on ordonne que l'on ordonne. Autrement dit, il s'agit là d'une efficacité de la parole, qui peut bénéficier à ce titre d'une analyse linguistique, puisqu'il n'y a d'action performative que s'il y a un énoncé à l'origine. Peut-on alors considérer que l'économie, de la même façon, crée certaines choses - et lesquelles - au moyen de la parole même, ou du discours de ceux qui l'énoncent? Et, surtout, comment comprendre que la parole fasse ces choses ?

On sait bien que la sociologie de la performativité admet s'être inspirée d'Austin pour ensuite s'en éloigner, en intégrant le discours parmi d'autres éléments sociotechniques comme conditions de la performativité. Reste qu'on peut alors s'interroger sur la légitimité ou l'intérêt du recours à une notion entée sur le discours et qui, à l'origine, n'a un sens que parce qu'elle permet d'identifier un phénomène jusque-là passé sous silence. Que gagne-t-on conceptuellement à qualifier de «performatifs » des effets de la théorie économique s'il s'agit simplement d'y voir les effets obtenus au moyen de dispositifs sociotechniques - c'est-à-dire des effets produits d'une façon qui, d'un point de vue conceptuel, n'a jamais vraiment été mystérieuse ? Autrement dit, on ne se débarrasse pas de l'enjeu conceptuel de l'usage du concept en considérant simplement qu'on le définit autrement : encore faut-il que cette redéfinition ait un sens et soit motivée. Pourquoi parler de "performativité » du discours économique si, par là, il ne s'agit plus de penser l'effet performatif obtenu au moyen du discours, mais les simples conséquences qui résultent de la mise en œuvre d'un discours économique donné au travers de dispositifs sociotechniques qui ont précisément pour objet de modifier 
le cours du monde et d'obtenir certains changements ? En quoi, pour le dire autrement, parle-t-on encore d'un effet du symbolique sur un réel qui ne l'est pas? S'il s'agit de dire qu'on ne le peut qu'en inscrivant le discours dans un ensemble de dispositifs qui lui permettent d'avoir une efficacité, on change d'approche - mais de ce fait, on ne parle plus vraiment des mêmes phénomènes et, dès lors, l'usage du terme de «performativité » risque d'être inadéquat, si ce n'est trompeur "s. Ainsi, s'il s'agit d'expliquer que la mise en œuvre de certains processus de calcul (construits par la théorie économique standard, par exemple le modèle Black-Scholes) conduit les agents économiques qui les utilisent à se comporter comme le prédit la théorie économique qui en est à l'origine, pourquoi parler de «performativité », alors que les effets produits semblent bien plus relever des effets «perlocutoires », ces effets produits par des moyens naturels qui n'ont pas besoin du discours pour advenir "19 ? Ne fait-on pas appel à un concept précisément construit (et légitime) pour penser autre chose ? Ne court-on pas alors le risque de confondre deux modalités d'efficacité, sans nier la pertinence propre de l'identification des effets de la science économique mis au jour par ladite «approche par le performatif »?

L'interrogation s'impose d'autant plus que l'enjeu de l'identification des conditions matérielles de cette efficacité du discours n'est pas nouveau. Déjà Austin, puis John Searle ou Pierre Bourdieu avaient dégagé des conditions non purement linguistiques, mais contextuelles et institutionnelles qui déterminent l'efficacité des énoncés performatifs. En ce sens, ils ont essayé de montrer que l'efficacité performative avait des conditions «matérielles » et «sociales » de réalisation. Et on peut dès lors être tenté de dire que l'économie, en tant qu'elle constitue bien un discours, de la même façon, se donne parfois les moyens de se mettre en œuvre ou de se réaliser performativement, que ce soit au plan

18. C'est déjà l'objet de la critique faite par Judith BUTLER à l'encontre de l'approche de Michel Callon : «Performing Agency », Journal of Cultural Economy, vol. 3, $\mathrm{n}^{\circ} 2$, p. 147-161.

19. Puisqu'on pourrait très bien imaginer que les mêmes effets (économiques) puissent être produits sans que les équations aient jamais été formulées dans un langage. Cela pose d'ailleurs la question du statut particulier du langage mathématique : peut-on vraiment considérer qu'il agit comme les énoncés performatifs ? En bonne rigueur austinienne, les énoncés de la mathématique doivent plutôt être considérés comme des énoncés ayant une vocation descriptive, c'est-à-dire comme étant susceptibles d'être évalués en termes de vérité ou de fausseté (dans l'ordre formel des mathématiques). C'est même tout ce qui fait leur intérêt. 
macroéconomique (politique économique) ou microéconomique (organisation des entreprises). L'économie comme science ou comme modèle (economics) trouverait ainsi, sous certaines conditions, à se réaliser dans l'économie comme domaine de la réalité sociale (economy). Reste que l'effet propre de l'efficacité performative est considéré par Austin (ou Searle, ou Bourdieu) comme un effet singulier: il s'agit proprement, pour reprendre les distinctions ultérieures, de l'effet illocutoire, distinct de l'effet perlocutoire, lequel s'apparente pour sa part plus aux simples conséquences d'un acte. L'effet illocutoire intervient logiquement du fait même de dire quelque chose, ou du moins certaines choses, dans certaines conditions. Ainsi, pour reprendre un exemple classique : léguer, c'est proférer un énoncé au moyen duquel, dans les bonnes conditions, le locuteur va agir d'une certaine façon en léguant quelque chose à quelqu'un. L'acte illocutoire produit réside dans le fait même de dire, même si cette réalisation est déterminée par autre chose que le simple langage ${ }^{20}$. Et cet acte consiste bien à opérer une modification dans l'ordre du monde : une fois le legs énoncé, quelque chose de nouveau existe dans le monde : un legs. C'est ce type de réalité que désigne l'effet illocutoire. Il existe plusieurs façons de la qualifier en termes de réalité symbolique ${ }^{21}$, de réalité déontique ${ }^{22}$, etc. - mais il s'agit toujours de la distinguer des effets produits perlocutoirement, c'està-dire par des moyens naturels qui ne nécessitent pas de manière essentielle le langage. Ainsi, il peut s'ensuivre du legs une joie profonde chez le futur héritier ou un dépit chez ceux qui sont déçus. Mais ces deux sentiments, qui sont bien produits par l'action de langage du legs, auraient très bien pu être produits autrement, sans passer par un énoncé. Pour le dire autrement, la joie produite comme un effet de l'acte de langage du legs n'est pas produite directement par l'énonciation, mais par les modalités de sa réception chez le destinataire. Elle n'est donc pas un effet performatif. Autant d'éléments qui font que subsiste dans certains cas une part d'incertitude, y compris descriptive, sur ce qui est pré-

20. Outre les textes d'Austin lui-même, voir sur ces points Bruno Ambroise, Qu'estce qu'un acte de parole?, Paris, Vrin, 2008 ; Marina SBISA, «Locution, illocution, perlocution », in Marina SBISA et Ken TURNER (eds.), Pragmatics of Speech Actions, Berlin, Mouton De Gruyter, 2013, p. 25-75.

21. C'est la solution de Pierre Bourdieu, Méditations pascaliennes, Paris, Seuil, 1997 ; Sur l'État, Paris, Raisons d'agir/Seuil, 2012.

22. C'est la solution développée par Marina Sbisà, l'éditrice d'Austin, qui rejoint en cela les propositions naguère faites par A. Reinach : voir notamment Marina SBISA, « On Illocutionary Types », Journal of Pragmatics, ${ }^{\circ}$ 8, 1984, p. 93-112. 
cisément visé, même une fois substitué «performation» à «performativité ».

\section{De quoi la «performation » est-elle le nom? (ou : ambivalence de la critique de la critique)}

On a suggéré plus haut que l'approche par la «performativité » avait le mérite d'éviter plusieurs écueils, parmi lesquels les divisions instituées par la théorie économique standard entre économie positive ou pure et économie normative ou appliquée ; divisions qui avaient déjà du plomb dans l'aile, d'autant que, pressés par la conjoncture, même des économistes guère connus pour leur goût immodéré de l'hétérodoxie se sont appliqués à démontrer qu'une théorie peut modeler le réel selon ses principes ${ }^{23}$. Au-delà, cette approche se démarque sans équivoque de toute forme de naturalisme (cognitivisme inclus) et, en cela au moins, elle produit des effets critiques, bienvenus à l'heure du succès croissant - et particulièrement préoccupant - d'un paradigme comme celui de la neuroéconomie. Du reste, ce courant se réfère volontiers à des auteurs fondateurs des sciences sociales, sans forcément marquer de distance avec les représentants de diverses traditions critiques ${ }^{24}$.

Reste qu'on ne peut s'empêcher de soulever à nouveau cette question de la critique qui, comme l'ont relevé M. Callon et F. Muniesa euxmêmes, est de fait au cœur des débats suscités par cette approche. Ses représentants semblent en effet naviguer entre plusieurs positions, prenant inégalement leurs distances vis-à-vis de traditions ouvertement critiques de l'économie et, plus généralement, de l'ordre social. Dans le chapitre du Traité de sociologie économique, les auteurs disent trouver les critiques épistémologiques et politiques existantes «frustrantes car elles se dispensent de regarder de manière empirique comment fonctionnent ces savoirs et comment ils affectent les pratiques économiques », avant d'expliciter le fait que le «vocabulaire de la performativité [...] préserve un certain nombre de préoccupations qui sont au cœur de la critique... ${ }^{25}$.

23. Par exemple Henri BourguInAT et Éric BRIYs, L'arrogance de la finance. Comment la théorie financière a produit le krach, Paris, La Découverte, 2009 ; et, par comparaison (d'avant la crise financière de 2007-2008), Donald MAcKenzIE, An Engine, not a Camera. How Financial Models Shape Markets, Cambridge, MIT Press, 2006.

24. Voir par exemple les mentions à Marx, Foucault, Lyotard ou Bourdieu in Fabian Muniesa et Michel CALlON, «La performativité des sciences économiques », op. cit., p. 293-295.

25. Ibidem, p. 318-319. 
L'accent est alors mis sur la continuité, au prix d'un surcroît d'exigence empirique et d'un droit d'inventaire (qui serait à préciser) quant aux «préoccupations » qui animent le regard critique. Ailleurs, à l'inverse, les flèches semblent prioritairement décochées à l'encontre de toute perspective se présentant ouvertement comme «critique », parfois même sur le mode de l'incompréhension surjouée ${ }^{26}$, tandis qu'inversement domine une impression d'indulgence (coupable) à l'égard du paradigme néoclassique en tant que tel. Cette ambivalence se retrouve notamment dans le rapport à l'auteur de La grande transformation: tantôt c'est la rupture qui est accentuée, sous la forme d'un renversement visant à "libérer du concept d'encastrement (embeddedness) »" tantôt, à l'inverse, c'est un complément ou un prolongement qui est proposé, avec l'idée que se trouve comblée une lacune théorique de l'héritage polanyien ${ }^{28}$. Certes, le réinvestissement de la notion de performativité dépend d'une perspective théorique qui se déploie sur un plan propre et, à vouloir à tout prix confronter des approches qui ne se rencontrent pas vraiment ou pas toujours - un travers des plus courants dans les joutes intellectuelles - on se condamne au dialogue de sourds. Néanmoins, l'altérité est aussi en partie une forme de rivalité et l'on se demande parfois si l'on n'a pas affaire à un affadissement sinon un anéantissement de perspectives analytiques qui s'exprimaient auparavant dans une autre terminologie, plus offensive à l'égard de l'ordre institué ou simplement, à un niveau plus modeste, de la théorie économique la plus standard.

On a, au passage, parfois l'impression que les tenants de cette démarche, dans leur volonté de se démarquer des approches plus ou moins constituées (par les conventions, les institutions, les structures, les propriétés sociales des acteurs, etc.), vont parfois un peu vite en besogne. Ainsi, l'idée selon laquelle « au-delà de sa polysémie, cette notion [d'encastrement] implique la même hypothèse, celle de la primauté des rapports sociaux sur les rapports économiques (primauté qu'affirment également les sociologies qui critiquent la notion d'encastrement pour lui pré-

26. Voir par exemple, sur l'analyse en termes d'idéologie, Michel CALLON, « Elaborating the notion of performativity », in Le Libellio d'AEGIS [en ligne], vol. 5, n 1, 2009, p. 19.

27. «Le choix de la notion d'agencement marchand comporte pour première conséquence de libérer du concept d'encastrement (d'embeddedness)» écrit Michel Callon dans sa postface à Franck CocHOY (dir.), Du lien marchand. Comment le marché fait société, Toulouse, PUM, 2012, p. 344.

28. Franck Cochoy, Martin GiRAudeau and Liz McFall, «Performativity, Economics and Politics », Journal of Cultural Economy, vol. 3, n 2, 2010, p. 141. 
férer celles de champs ou d'institutions) » ${ }^{29}$ aligne de façon quelque peu réductrice des adversaires bien distincts. De ce point de vue, la démarcation vis-à-vis de traditions critiques fort diverses, au nom du pluralisme contre le mécanisme ${ }^{30}$, laisse sceptique. Un sentiment accentué à l'aune de l'ambition intellectuelle bien décevante consistant par exemple à " comprendre ce qu'est un "bon" marché » "s, sans égard pour la critique des rapports marchands, sans parler de la marchandisation des rapports sociaux. Force est alors de constater que, de manière plus ou moins explicite, ce système théorique évince de fait un certain nombre de concepts qui, tels «économisme », «idéologie », «fétichisme », « réification » ou «pouvoir symbolique», étaient et demeurent associés à une critique théorique plus ou moins assumée de l'ordre économique et social et ce à travers l'analyse des rapports sociaux non seulement matériels, mais aussi symboliques. Il n'est pas dit que l'on y gagne nécessairement au change, y compris d'un point de vue intellectuel général délié d'une intention critique explicite.

Une rapide confrontation avec Marx, Bourdieu et Foucault (pour s'en tenir là) invite à la fois à relativiser les apports de l'approche par le performatif et à repérer les articulations possibles avec d'autres perspectives. Ceci afin de mettre en relief, parfois derrière des effets de slogan ou d'affichage, des lignes de continuité dans le travail réflexif des sciences sociales. C'est la raison pour laquelle, explicitement ou implicitement, les liens entre performativité et idéologie, ou performativité et pouvoir symbolique, traversent les contributions réunies dans ce dossier. On peut d'abord se demander si la notion de «performativité » (de même que celle de performation), telle qu'elle est utilisée par l'approche performativiste, n'affaiblit pas la critique des effets de domination du discours économique, notamment par rapport à la notion d'origine marxienne d' «idéologie », construite et mobilisée au sein d'une perspective structurale globale sur le mode d'être des humains vivant en société :

«Dans la production sociale de leur existence, les hommes nouent des rapports déterminés, nécessaires, indépendants de leur volonté ; ces rapports de production correspondent à un degré donné du développement de leurs forces productives matérielles. L'ensemble de ces rapports forme la structure économique de la société, la fondation réelle sur laquelle s'élève un édifice juridique et poli-

29. Michel CALLON, postface à Franck CoCHOY (dir.), Du lien marchand, op. cit., p. 344-345.

30. Comme le suggère Jérôme Denis en conclusion de son article de 2006 cité supra.

31. Michel Callon, postface à Franck Cochoy (dir.), Du lien marchand, op. cit., p. 351 . 
tique, et à quoi répondent des formes déterminées de la conscience sociale. Le mode de production de la vie matérielle domine en général le développement de la vie sociale, politique et intellectuelle. Ce n'est pas la conscience des hommes qui détermine leur existence, c'est au contraire leur existence sociale qui détermine leur conscience. ${ }^{32}$ »

Par idéologie, on entend un discours qui, tout en prétendant décrire un état de fait, vient en fait après coup masquer, dissimuler et déformer tel ou tel domaine de la réalité sociale pour les acteurs qui la vivent - à commencer par l'exercice d'une domination de classe ${ }^{33}$. Depuis lors au moins, il est établi que le discours économique n'est pas seulement descriptif (quand bien même il pourrait avoir des effets de distorsion de la réalité et produirait une illusion), mais aussi normatif et parfois prescriptif. Il est une force qui informe et transforme les pratiques et les représentations sociales, et tend à façonner la réalité comme «le modèle le veut » ou à évaluer cette réalité à l'aune de ce modèle qui lui fixe ce qu'elle a désormais à être. Le devoir-être n'est donc pas un simple habillage de l'apparence pour la légitimer et reproduire un état de chose ; il entend reconfigurer l'être social en profondeur. Cette dimension «constructiviste» que les travaux actuels sur la «performativité du discours économique » entendent mettre au jour était-elle complètement absente du concept «classique » d'idéologie ? Réciproquement, la notion de performativité ici déployée rend-t-elle compte de tout ce dont envisageait de rendre compte la notion d'idéologie ? Ne fonctionne-t-elle pas comme une sorte d'euphémisme destiné à adoucir la portée en termes de critique sociale que l'on trouve dans les termes d'idéologie, de fétichisme, d'économisme ou de réification - concepts qui n'ont probablement pas dit leur dernier mot ? ${ }^{34}$

On peut alors se demander en quoi l'usage d'une acception élargie du concept de performativité est plus heuristique qu'un autre concept, celui de «pouvoir symbolique » élaboré par Pierre Bourdieu, qui lui-même reprenait à son compte les découvertes austiniennes sur l'efficacité du langage. Il désignait par là, résumé au plus simple, une forme de pouvoir

32. Karl MARX, «La critique de l'économie politique », in Euvres - Économie, 1, Gallimard, « La Pléiade », 1963, p. 272-273.

33. Pour plus de développement, voir Richard SoBEL, «Idéologie, sujet, subjectivité en théorie marxiste. Marx et Althusser », Revue de philosophie économique, 14 (2), 2013, p. 151-192

34. Pour une reprise collective récente de ce dernier concept, voir Vincent CHANSON, Alexis CukiER et Frédéric Monferrand (dir.), La réification. Histoire et actualité d'un concept critique, Paris, La Dispute, 2014. 
méconnue comme telle et qui fonctionne ainsi d'autant plus facilement et efficacement. Or, il l'utilisait précisément pour rendre compte de phénomènes plus larges que la simple performativité linguistique, tout en l'incluant ${ }^{35}$. Pour lui, en appeler à l'idée d'une force illocutoire autonome était elle-même illusoire et typique d'un mouvement de pensée essentialiste et idéaliste, niant la détermination sociale de l'efficacité symbolique :

«Le pouvoir symbolique, pouvoir de constituer le donné en l'énonçant, d'agir sur le monde en agissant sur la représentation du monde, ne réside pas dans les "systèmes symboliques" sous la forme d'une "force illocutionnaire". Il s'accomplit dans et par une relation définie qui crée la croyance dans la légitimité des mots et des personnes qui les prononcent et il n'opère que dans la mesure où ceux qui le subissent reconnaissent ceux qui l'exercent. ${ }^{36}$ »

En développant ainsi une véritable analyse de l'efficacité symbolique, Bourdieu proposait bien plutôt une explication de la force illocutoire qui la reconduisait à ses déterminants sociaux et évitait d'en faire la composante mythique d'un pouvoir inexpliqué.

«Si, comme le remarque Austin, il est des énonciations qui n'ont pas seulement pour rôle de "décrire un état de choses ou d'affirmer un fait quelconque", mais aussi d" "exécuter une action", c'est que le pouvoir des mots réside dans le fait qu'ils ne sont pas prononcés à titre personnel par celui qui n'en est que le "porteur": le porte-parole autorisé ne peut agir par les mots sur d'autres agents [...] que parce que sa parole concentre le capital symbolique accumulé par le groupe qui l'a mandaté et dont il est le fondé de pouvoir. [...] Un énoncé performatif est voué à l'échec toutes les fois qu'il n'est pas prononcé par une personne ayant le "pouvoir" de le prononcer ou, plus généralement, toutes les fois que "les personnes ou circonstances particulières" ne sont pas "celles qui conviennent pour qu'on puisse invoquer la procédure en question", bref toutes les fois que le locuteur n'a pas d'autorité pour émettre les mots qu'il énonce. ${ }^{37}$ »

C'est dans cette perspective, probablement plus fidèle à la compréhension austinienne de l'efficacité performative dont elle propose un autre usage ${ }^{38}$, que s'inscrit la contribution de Frédéric Lebaron dans ce dossier, intitulée «Discours d'institution et pouvoir symbolique : le cas des banques centrales », à partir d'une analyse du discours de la Banque centrale européenne.

35. Voir Pierre Bourdieu, Langage et pouvoir symbolique, Paris, Seuil, 2001.

36. Pierre BouRdieu, Raisons pratiques, Paris, Seuil, 1994, p. 188.

37. Pierre Bourdieu, Langage et pouvoir symbolique, op . cit., p. 60.

38. Voir Bruno Ambroise, «Une conception non scolastique de l'efficacité linguistique : Bourdieu lecteur d'Austin », in Marie-Anne Lescourret (dir.), Pierre Bourdieu, un philosophe en sociologie, Paris, PUF, 2009, p. 65-88. 
Par ailleurs, le rapprochement entre les analyses en termes de «performativité/performation» et celles antérieures de Michel Foucault (et Gilles Deleuze) a été effectué via des développements autour de l'importation des notions d'agencement et de dispositif ${ }^{{ }^{3}}$. À cet égard, il convient de rappeler qu'on peut trouver chez l'auteur de Surveiller et punir des développements précisément consacrés à la façon dont idées et discours façonnent le réel, médiations techniques incluses — par exemple la prison, dispositif par excellence, qui articule du discursif et du non discursif. Mais l'on peut aussi penser, dans un autre genre, à l'analyse de l'ordolibéralisme (la branche allemande du néolibéralisme) qu'il proposait à la fin des années $1970^{\circ 0}$. On y trouve bien, en effet, une analyse de la façon dont une doctrine, personnifiée par un certain nombre de théoriciens et notamment d'économistes, fonde non seulement les principes de fonctionnement d'une configuration du capitalisme appelée « économie sociale de marché », mais encore comment celle-ci est ellemême fondatrice d'une nouvelle souveraineté politique qui ne pouvait procéder directement de l'État, vu le discrédit jeté sur cette figure après le désastre nazi. Plus largement, on est même tenté de se demander si le concept de gouvernementalité, comme art de conduire les conduites par des moyens aussi bien discursifs que matériels, n'englobe pas, d'une certaine manière, ce que l'on cherche à saisir avec celui de performativité (ou de performation).

Il est certain en tout cas que la rationalité néolibérale qui régit les sociétés capitalistes avancées commande une analyse serrée des conditions intellectuelles et institutionnelles ainsi que des canaux multiples (le cas échéant prosaïquement matériels, en apparence du moins) à travers lesquels l' «économie » tend à régler, symboliquement comme matériellement, de multiples domaines de la vie sociale. C'est, entre autres, le cas de la santé, étudié par Philippe Batifoulier dans sa contribution à ce dossier («Performativité et théorie conventionnelle: une application à l'assurance santé »), secteur dont il montre qu'il est typique de la «fabrication sociale de la réalité conforme au raisonnement économique ». Il en va de même de celui desdits « services à la personne », dont rend compte Florence Jany-Catrice («La légitimation académique d'un projet politique :

39. Voir par exemple Hervé Dumez et Alain Jeunemaitre, «Quand l'économie échoue à être performative », in Madeleine AKRICH et al. (dir.), Débordements, op. cit., p. 129-141.

40. Michel Foucault, Naissance de la biopolitique. Cours au Collège de France 1978-1979, Paris, Gallimard/Seuil/EHESS, 2004. 
la création des "services à la personne" ") en pointant les effets d'une combinaison de dogmatisme et de pragmatisme. Au-delà des cas d'étude, c'est sans doute parce que ce qui reconfigure le plus profondément le mode de subjectivation aujourd'hui est le discours néolibéral et sa promotion-diffusion-extension de l'homme économique que la question de la «performativité de l'économie» occupe une place centrale. C'est à une réflexion de ce type, épistémologique autant que politique, qu'invite François Flahault dans son article "Quand la science contribue à créer l'ordre du monde ». À ce niveau, la démarche constructiviste est radicale et fait fond sur une réflexion anthropologique concernant le modèle de l'homo ceconomicus ou de l'homme performant, réflexion en quelque sorte appelée par l'état de «crise » permanent qui sert de cadre discursif à l'époque présente.

Cette «crise » qui, on l'a dit en introduction, a suscité une masse inhabituelle de réflexions consacrées à la contribution des errements de la science économique dominante, met à l'épreuve, à sa manière, les apports de la sociologie économique issue de la théorie de l'acteur-réseau. Or, de ce point de vue, la déception l'emporte. C'est en quelque sorte au moment où l'on aurait le plus besoin des analyses qu'elle est susceptible de fournir que cette approche semble se dérober, du moins dans l'espace intellectuel public. L'enjeu a certes été soulevé "t mais, en dépit de quelques textes d'intervention de Donald MacKenzie notamment, la moisson semble fort maigre, comme si les tenants de cette approche ne se sentaient pas requis par la situation présente. Quand ce dernier analyse, en 2011, la crise du crédit en tant que problème pour la sociologie de la connaissance, où la notion de performativité et ses dérivés pourraient $a$ priori trouver leur place, il n'y recourt pas ${ }^{42}$. De son côté Fabian Muniesa, dans son livre de 2014 déjà cité, The Provoked Economy, dont Bruno Ambroise propose ici une lecture critique, fait l'impasse sur le sujet. Qu'il s'agisse là de textes académiques n'est pas anodin et témoigne du fait que si le succès de l'approche ici considérée ne fait aucun doute, il reste pour l'essentiel cantonné à la sphère universitaire. À l'inverse, des contributions non seulement spécialisées mais destinées à un public élargi ont afflué du côté de l'hétérodoxie économique et de la théorie sociale, aussi bien de la part de l'école de la régulation que de diverses

41. Franck Cochoy, Martin GiRaudeau et Liz McFall, « Performativity, Economics and Politics », art. cit., p. 139-146.

42. Donald MacKenzie, «The Credit Crisis as a Problem in the Sociology of Knowledge », American Journal of Sociology, vol. 116, n 6, 2011, p. 1778-1841. 
sensibilités marxistes en passant par les post-keynésiens et des héritiers de Bourdieu ; parfois avec une force incisive salutaire, comme c'est le cas du livre de Philip Mirowski Never Let a Serious Crisis Go to Waste présenté ici par Grégory Salle. Sans forcément parler d'une faillite de la sociologie de la traduction en la matière, force est de constater que nous avons encore fort besoin des traditions critiques qu'elle tend à écarter.

Bruno Ambroise (CNRS, CURAPP-ESS)

Grégory Salle (CNRS, CLERSÉ)

Richard Sobel (Université Lille 1, CLERSÉ)

* * * $*$ 